\title{
Analytic Solutions for the Antigreenhouse Effect: Titan and the Early Earth
}

\author{
Christopher P. McKay \\ Space Science Division, NASA Ames Research Center, Moffett Field, California 94035 \\ Email: cmckay@mail.arc.nasa.gov \\ and \\ Ralph D. Lorenz and Jonathan I. Lunine \\ Lunar and Planetary Laboratory, University of Arizona, Tucson, Arizona 85721
}

Received April 27, 1998; revised September 14, 1998

\begin{abstract}
Theantigreenhouse effect resultsfrom a high altitude haze which blocks sunlight while allowing transmission of thermal infrared. An antigreenhouse is observed on Titan and has been proposed for the early $E$ arth. Here, we derive an analytic solution useful for computing the atmospheric and surface temperature with both the greenhouse and antigreenhouse effects included. In agreement with detailed calculations, the analytic solution predicts that a runaway greenhouse on early Titan caused by accretional heat would collapse when the flux fell to $0.9 \mathrm{~W} \mathrm{~m}^{-2}$. For the present value of the solar luminosity a runaway greenhouse collapses at a geothermal heat flux of $0.4 \mathrm{~W} \mathrm{~m}^{-2}$. We show that the antigreenhouse effect may compromise attempts to solve the faint young sun problem on the early $E$ arth with a methane-ammonia greenhouse shielded by an organic haze, more than doubling the infrared opacity needed to warm the early Earth compared to the case with no antigreenhouse. (c) 1999 Academic Press
\end{abstract}

Key Words: Titan; atmosphere; greenhouse effect; antigreenhouse effect.

\section{INTRODUCTION}

The greenhouse effect arises because certain atmospheric gases are transparent to sunlight but are opaque to thermal infrared. On Earth, Mars, and Venus, $\mathrm{CO}_{2}$ and $\mathrm{H}_{2} \mathrm{O}$ are the principal greenhouse gases. By contrast, Titan has a strong greenhouse effect due to $\mathrm{N}_{2}, \mathrm{CH}_{4}$, and $\mathrm{H}_{2}$ (Samuelson 1983, McKay et al. 1989, 1991). Titan also has an antigreenhouse effect caused by a high altitude organic haze layer (McKay et al. 1991). Titan's haze is opaque at visible wavelengths but is virtually transparent to thermal infrared wavelengths. Thus, it blocks sunlight from reaching the surface but readily allows thermal radiation to escape. Detailed numerical simulations have quantified the greenhouse and antigreenhouse effects on Titan - the greenhouse raises the surface temperature by $21 \mathrm{~K}$ while the antigreenhouse reduces it by
$9 \mathrm{~K}$ (McKay et al. 1991). The variation of these effects over geological time and solar luminosity has been investigated (McKay et al. 1993, Lorenz et al. 1997a, 1997b).

Recently Sagan and Chyba (1997) have proposed that the early Earth also had an organic haze layer produced, as on Titan, by $\mathrm{CH}_{4}$ photolysis. Such a layer would preferentially absorb ultraviolet light thereby allowing $\mathrm{NH}_{3}$ to persist in the atmosphere. The strong greenhouse which results from the combination of $\mathrm{CH}_{4}$ and $\mathrm{NH}_{3}$ would then be adequate to offset the faint young sun. However, we suggest that the antigreenhouse effects of such a haze layer could negate any greenhouse warming.

In this paper we develop an analytic solution for the atmospheric temperature profile for the combined greenhouse and antigreenhouse effects in the gray case (opacity uniform with wavelength). We compare the results from this simple representation with detailed numerical simulations and use it to examine the stability of Titan's climate. We also consider how the antigreenhouse effect of a haze layer would offset the greenhouse effect on the early Earth.

\section{ANTIGREENHOUSE GRAY CALCULATION}

The surface temperature of a planet considering only a gray greenhouse effect is given by

$$
T_{\mathrm{s}}^{4}=T_{\mathrm{e}}^{4}\left(1+\frac{3}{4} \tau^{*}\right)
$$

where $T_{\mathrm{s}}$ is the surface temperature, $\tau^{*}$ is the column infrared grey opacity, and $T_{\mathrm{e}}$ is the effective temperature given by $\sigma T_{\mathrm{e}}^{4}=$ $F_{\mathrm{s}}$ where $\sigma$ is the Stefan-Boltzman constant and $F_{\mathrm{s}}$ is the globally averaged solar flux absorbed by the surface and atmosphere. Equation (1) is inadequate for Titan because of the antigreenhouse effect. However, Samuelson (1983) has shown that Titan's temperature profile can be realistically simulated with a 
three component radiative scheme. The three components are: (1) solar flux deposited on the surface, (2) gray infrared cooling, (3) solar flux absorbed in the stratosphere. The greenhouse effect arises from the first two and the antigreenhouse effect from the third.

Equation (1) can be generalized to include this antigreenhouse effect. As described in the Appendix, we obtain a simple expression for the temperature in the atmosphere and at the surface. In the antigreenhouse limit the surface temperature is given by

$$
\sigma T_{\mathrm{s}}^{4}=\left(F_{\mathrm{g}}+(1-\gamma) F_{\mathrm{s}}\right)\left(1+\frac{3}{4} \tau^{*}\right)+\frac{\gamma F_{\mathrm{s}}}{2}
$$

where $F_{\mathrm{g}}$ is a geothermal heat term added for generality, $F_{\mathrm{s}}=$ $(1-A) F_{0} / 4$, where $F_{0}$ is the solar constant at Titan's heliocentric distance, $A$ is the albedo, and $\gamma$ is the factor expressing the strength of the antigreenhouse effect. Physically $\gamma$ is the fraction of sunlight, not reflected to space, that is blocked by the antigreenhouse layer; the sunlight reaching the lower atmosphere is therefore $(1-\gamma)(1-A) F_{0} / 4$.

\section{PARAMETERS FOR ANTIGREENHOUSE EQUATION FOR TITAN}

For the present Titan the values of the parameters needed to compute the surface temperature can be obtained by comparing the computations of the antigreenhouse equation (see Appendix) with the observed temperature structure on Titan (Lellouch et al. 1989) and the computed radiative fluxes in the atmosphere (McKay et al. 1989, 1991). Following Fig. 1 of McKay et al. (1991) we note that the incident sunlight averaged over the disk of Titan $\left(F_{0} / 4\right)$ is about $3.5 \mathrm{~W} \mathrm{~m}^{-2}$. Of this, 0.3 is reflected to space, 0.4 is absorbed in the stratosphere, and 0.3 reaches the lower atmosphere. Thus, the factor $\gamma$ is equal to $0.4 /(0.3+0.4)$. Note that in our approach we compute the greenhouse effect with all the flux reaching the troposphere $(0.3)$ and not just that reaching the surface (0.1). The solar flux absorbed by Titan's stratosphere composes the antigreenhouse effect. Of this fraction, 0.4 , of the solar flux, 0.25 is reradiated to space and 0.15 is reradiated toward the troposphere (McKay et al. 1991).

The total infrared opacity, $\tau^{*}$, and the parameter that determines the extinction in the antigreenhouse layer, $k$ (see Appendix), were determined by comparing the temperatures of the simple calculation at the surface, the tropopause, and at top of the atmosphere $(\tau \rightarrow 0)$ with the Voyager (Lellouch et al. 1989) results for these same locations. Our resulting values are $\tau^{*}=3$ and $k=160$. Thus we find that the effective opacity in the thermal infrared is only a few optical depths presumably due to radiation leaking though the large spectral window region between 300 and $600 \mathrm{~cm}^{-1}$ (McKay et al. 1989). This agrees in magnitude with the computed Rosseland mean infrared opacity for the present troposphere of about 2 . We find that the haze layer is strongly absorbing in the visible, in agreement with nongrey simulations (McKay et al. 1991).
In our simple parameterization of the antigreenhouse effect the ratio of optical to infrared opacity is assumed constant and large. This assumption is valid in Titan's stratosphere where virtually all of the antigreenhouse absorption is occurring.

The antigreenhouse parameterization gives the temperature profile in terms of the infrared optical depth throughout the atmosphere. To relate opacity to a physical variable we consider a power law representation between opacity, $\tau$, and pressure, $P$, given by $\tau \propto P^{n}$. To determine $n$ we compare the temperature dependence on altitude prescribed by the antigreenhouse equation (Eq. (7) in the Appendix) with the data for the present atmosphere. Figure 1 compares Titan's temperature profile with the vertical temperature profile computed for the present atmosphere $\left(F_{\mathrm{g}}=0, k=160, \tau^{*}=3, \gamma=4 / 7\right)$ for values of $n$ equal to $4 / 3$ and to 2 . The fit is quite good for $n=4 / 3$. There is a $3 \mathrm{~K}$ difference between the air temperature at the ground and

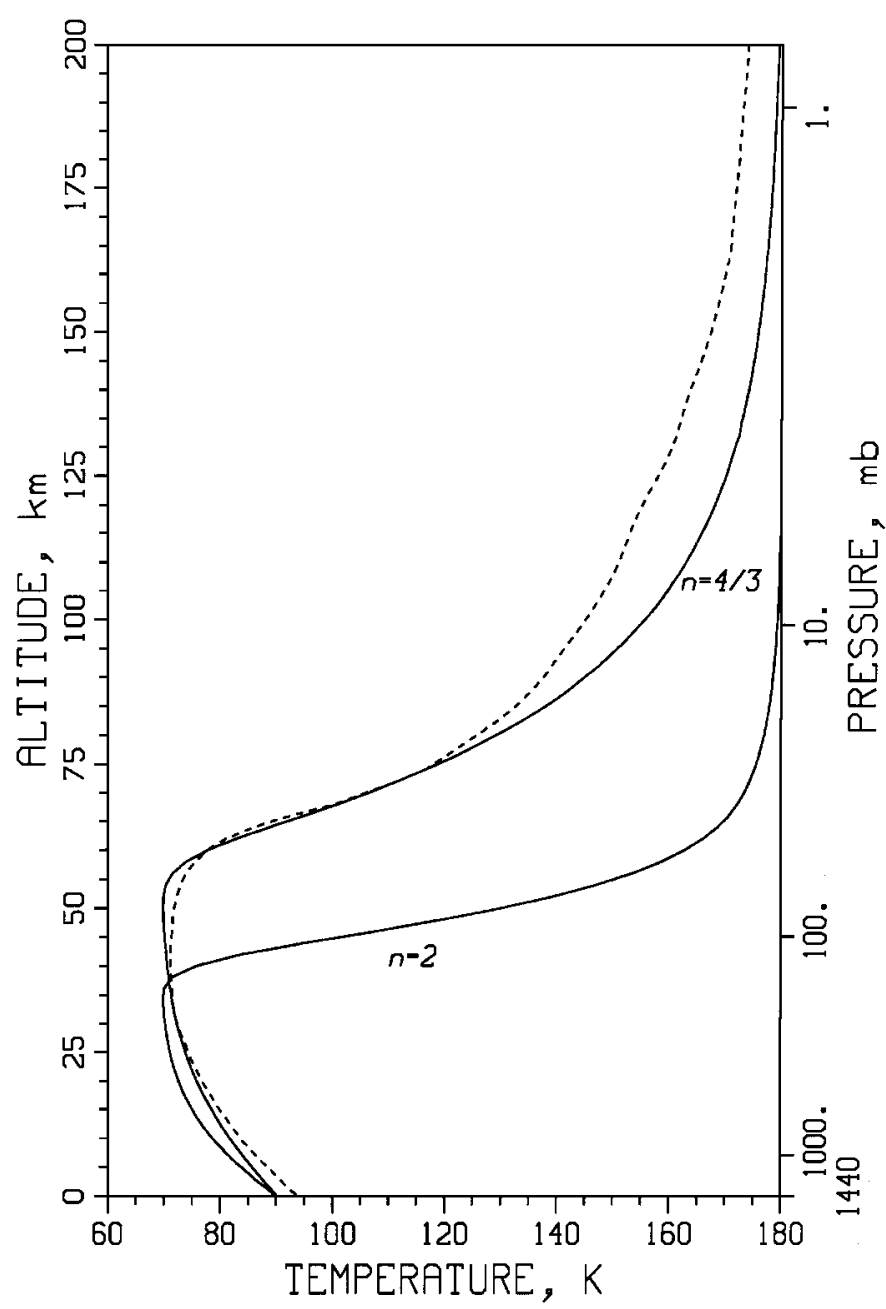

FIG. 1. Vertical profile determined from the analytical antigreenhouse (Eq. (7)) for opacity-pressure power laws of $n=2$ and $n=4 / 3 ; \tau^{*}=3, \gamma=$ $4 / 7, k=160$. The results for $n=4 / 3$ compare favorably with the present atmosphere determined by Lellouch et al. (1989) from Voyager 1 radio-occultation and other data (dashed line). 


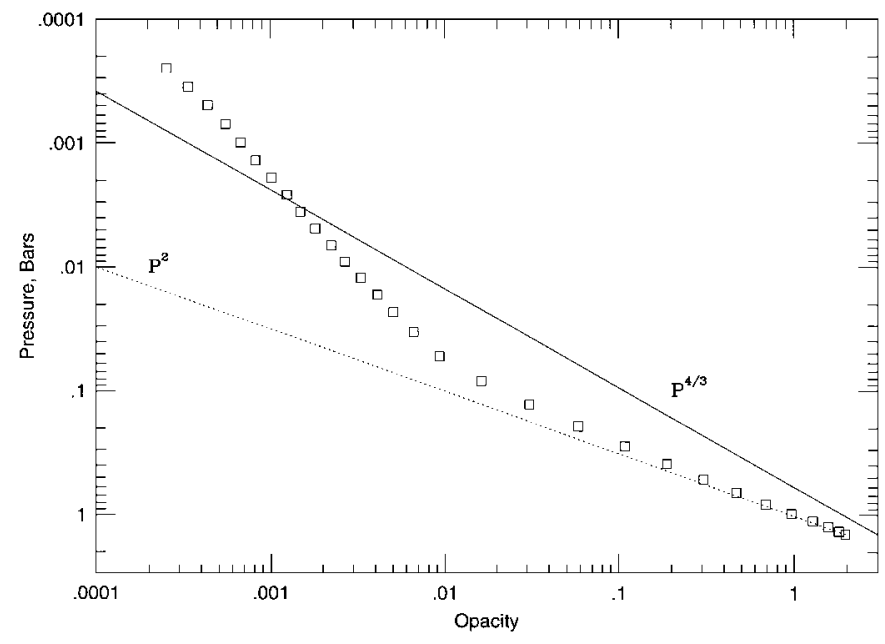

FIG . 2. Rosseland mean opacity determined for Titan's atmosphere compared to power laws of 2 and $4 / 3$.

the surface temperature due to the difference in radiative equilibrium temperatures between the surface and the bottom of the atmosphere (Eqs. (7) and (8) in the Appendix). The coefficients were selected to match the ground temperature. One might have expected $n=2$ to provide the best fit since the major source of infrared opacity in Titan's lower atmosphere is collision induced absorption which depends on density squared. However, above the troposphere the infrared opacity is dominated by the haze and the dependence on pressure is weaker. The net result is that the best overall fit is obtained with $n=4 / 3$. This is illustrated in Fig. 2 in which we have plotted the Rosseland mean opacity from the nongray case along with curves proportional to $P^{2}$ and $P^{4 / 3}$. Even in the troposphere the $4 / 3$ power law fits the temperature data (Fig. 1) better than $n=2$.

\section{APPLICATION TO TITAN}

Having developed the antigreenhouse equation for the present Titan we can test its ability to predict changes there. One interesting limit would be the case in which the antigreenhouse effect is completely removed. Detailed nongray numerical simulations (McKay et al. 1991) have found that with the haze removed the surface temperature rises from $94 \mathrm{~K}$ to $105 \mathrm{~K}$, and with $\mathrm{CH}_{4}$ stratospheric absorption also removed the surface temperature rises to $115 \mathrm{~K}$. In these runs coupling with any volatile reservoirs was not considered, i.e., the surface pressure was held constant at 1.44 bars. In our antigreenhouse formula this case is equivalent to holding $\tau^{*}$ constant while $\gamma \rightarrow 0$. The surface temperature computed with Eq. (2) is $110 \mathrm{~K}$, comparable to the value found in the nongray computation. For the obverse case of a complete antigreenhouse effect, $\gamma \rightarrow 1$, Eq. (2) gives the expected result (McKay et al. 1991): a surface temperature of $T_{\mathrm{e}} / \sqrt[4]{2}=71 \mathrm{~K}$.

We now consider the case of heat added to Titan as a way to explore the stability of the present climate state. We consider that the atmosphere is in equilibrium with surface reservoirs of
$\mathrm{CH}_{4}$ (lakes) but not a deep global ocean. For this comparison we set the total exchangeable reservoir of $\mathrm{CH}_{4}$ to 2 bars. We have computed the surface temperature for surface heat flows up to $1 \mathrm{~W} \mathrm{~m}^{-2}$ and for values of the solar constant from 0.7 to 1.6 times the present value (McKay et al. 1989). Our results are shown in Fig. 3 and are compared to the predictions of the analytic antigreenhouse equation. For surface temperature less than $100 \mathrm{~K}$, $\mathrm{CH}_{4}$ remains a minor constituent in the atmosphere and we let its supersaturation reach $140 \%$ as suggested by observations and theory for the present Titan (Courtin et al. 1995, Samuelson et al. 1997); these symbols are the squares in Fig. 3. Above $100 \mathrm{~K}$ $\mathrm{CH}_{4}$ becomes a major constituent and inhibition of condensation is less likely and so we set the limit of the humidity to $100 \%$. In all cases no clouds are present and the haze production rate and mixing ratio of $\mathrm{H}_{2}$ are held constant at their present level (McKay et al. 1989). The nongrey results show a collapse of the methane-rich atmosphere occurring when the geothermal heat reaches a value of $\sim 0.4 \mathrm{~W} \mathrm{~m}^{-2}$. For heat flows above this value all available $\mathrm{CH}_{4}$ is in the atmosphere and surface temperature increases smoothly with increasing heat flow. We have primarily considered the effects of cooling from an initial hot state since the primary application of interest is the end of the accretion phase on Titan.

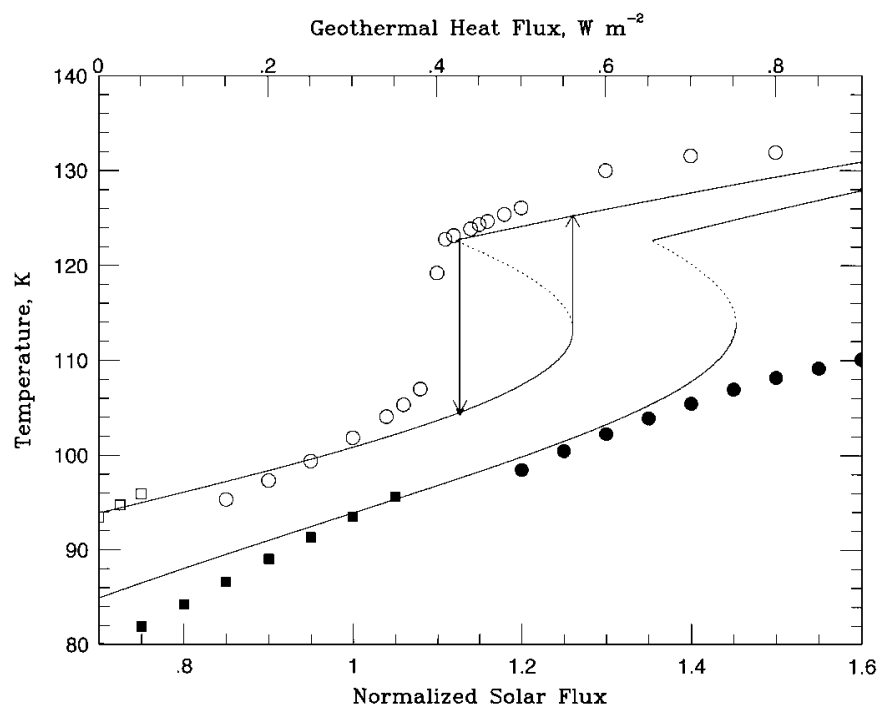

FIG . 3. Comparison of analytic solution with detailed calculation for added heat on Titan for lake-like reservoirs on the surface which contain a total of 2 bars of $\mathrm{CH}_{4}$. The temperature computed for the surface of Titan for added heat using the detailed nongray simulation (McKay et al. 1991) is shown by open symbols for geothermal heat $\left(F_{\mathrm{g}}\right)$ on the upper abscissa and shown by filled symbols for solar flux $\left(F_{\mathrm{S}}\right)$ normalized to the present value on the lower abscissa. Square points indicate cases in which $\mathrm{CH}_{4}$ is a minor constituent and is limited to $140 \%$ humidity. Circles represent cases in which $\mathrm{CH}_{4}$ is a major constituent and limited to $100 \%$ humidity. The lines are the results of the analytic antigreenhouse equation. The dotted lines show where the solution is unphysical due to the runaway greenhouse and the trajectory would be expected to jump from the lower trajectory to the upper line as shown by the arrows. Collapse from a warm state occurs in the detailed simulations and in the analytic solution at a geothermal flux of $0.4 \mathrm{~W} \mathrm{~m}^{-2}$. Total $\mathrm{N}_{2}$ is set at 1.3 bars. 
The result from the simple antigreenhouse formula is shown as the dotted and solid lines in Fig. 3. At each point the temperature was chosen and the pressure of $\mathrm{CH}_{4}$ determined and added to the constant pressure of $\mathrm{N}_{2}$. This pressure was used to compute the opacity from $\tau \propto P^{n}$, and the heat flow or solar insolation corresponding to that temperature was determined from Eq. (2). The dotted lines show regions that are mathematical solutions to the equation but have no physical significance. For geothermal heating levels between 0.4 and $0.6 \mathrm{~W} \mathrm{~m}^{-2}$ there are two physical solutions. The expected trajectory of the atmosphere with changing heat flow is shown by the arrows. The detailed simulations follow the warm branch of the solution since the iteration technique determines the temperature from initial values larger then equilibrium. The antigreenhouse equation accurately reproduces the collapse of the runaway greenhouse effect seen in the detailed simulation as geothermal heating is reduced. Considering the simplicity of the expression the agreement is good. Note that the parameters in the antigreenhouse equation were chosen to precisely fit the present conditions only $\left(F_{\mathrm{g}}=0 ; F_{\mathrm{s}}=1\right)$; no parameters were selected to fit the results at any other values of solar or geothermal heating.

Turning now to the solar flux, the results of the detailed calculations are in agreement with the antigreenhouse formula for values below 1.4. Above this level the detailed results are lower than the predictions of the antigreenhouse equation. The runaway predicted by the antigreenhouse equation at $110 \mathrm{~K}$ is not reached in the nongrey calculations. The reason for this is that in the nongray calculations the stratosphere expands as the solar heating increases and this results in a physically thicker haze layer even with the same haze production rate. Thus the antigreenhouse effect becomes larger as the solar flux increases. As the solar flux changes from 0.7 to 2.0 the antigreenhouse parameter increases by $35 \%$, as determined by the detailed nongray simulations. This increase in $\gamma$ effectively cools the atmosphere. Note that increasing surface temperature due to geothermal heat has no significant effect on the antigreenhouse parameter.

It is interesting to consider the case of early Titan with geothermal heating provided by accretional heating when the solar luminosity was $70 \%$ of its present level. The detailed simulations and the predictions of the antigreenhouse formula for this case are shown in Fig. 4. By alternately setting the initial conditions used in the nongray model to slightly warmer and slightly colder than the analytic results, we have been able to use the detailed model to compute both trajectories in the region of dual stable solutions. The results show the collapse of a hot methane-rich atmosphere occurs at a flux of about $0.85 \mathrm{~W} \mathrm{~m}^{-2}$ while values of $0.9 \mathrm{~W} \mathrm{~m}^{-2}$ are needed to cause runaway from a cold state. The antigreenhouse equation predicts the surface temperature and the asymmetric trajectories to within $5 \mathrm{~K}$ over the range of heat flows. This is noteworthy because the parameters in the antigreenhouse equation are chosen to give agreement with the present conditions. No parameters have been selected to give agreement with past conditions and this is therefore a realistic test of the utility of the method.

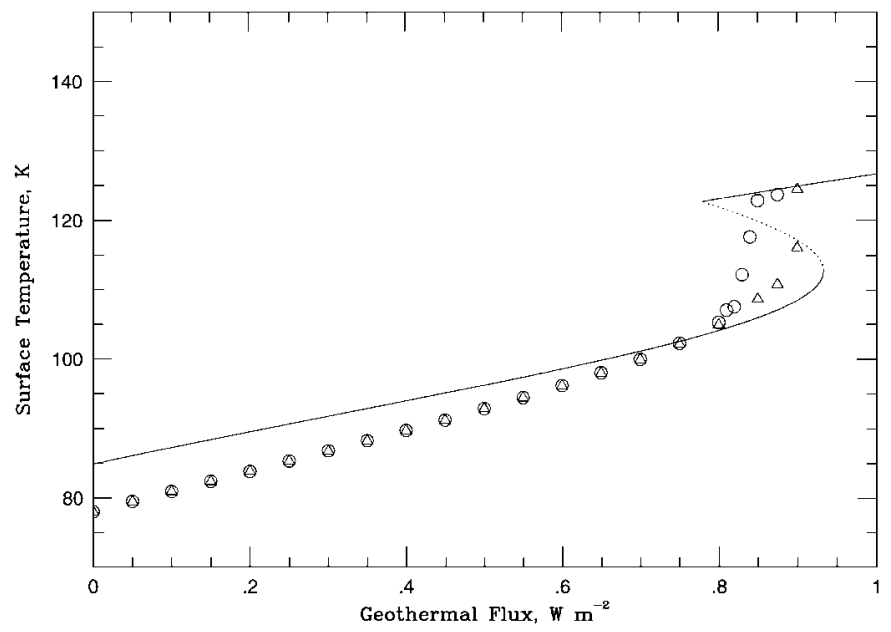

FIG . 4. Effect of surface heating, due to accretional heating, on Titan when the solar constant was 0.7 times its present value. The lines are the results of the analytic antigreenhouse equation. The dotted lines show where the solution is unphysical and the trajectory would be expected to jump from the lower trajectory to the upper line or vice versa. Symbols are from the detailed nongray calculation: triangles correspond to cold initial conditions and circles correspond to warm initial conditions. A runaway greenhouse occurs (triangles) at a temperature of $110 \mathrm{~K}$ and a surface heat flow of $0.9 \mathrm{~W} \mathrm{~m}^{-2}$. The total inventory of $\mathrm{CH}_{4}$ is assumed to be 2 bars, which limits the extent, but not the onset of the runaway. Greenhouse collapse (circles) occurs at heat flows of about $0.85 \mathrm{~W} \mathrm{~m}^{-2}$ and depends on the total methane assumed. Total $\mathrm{N}_{2}$ is set at 1.3 bars.

\section{APPLICATION TO THE EARLY EARTH}

We have developed and tested our simple antigreenhouse equation for the atmosphere of Titan because the antigreenhouse effect is well characterized for that world in both theory and observations. However, it has recently been suggested that there may have been an organic haze layer on the early Earth (Sagan and Chyba 1997) that absorbed ultraviolet sunlight. The ultraviolet shielding provides a mechanism for protecting photolabile compounds, particularly $\mathrm{NH}_{3}$ which can provide a strong greenhouse on the early Earth to offset the faint early sun. However, such a layer would also have an antigreenhouse effect.

For the case of Titan we have considered the opposite limits of no antigreenhouse effect $(\gamma=0)$ and a complete antigreenhouse effect $(\gamma=1)$ but photochemical and microphysical models are not adequate to predict how the antigreenhouse effect would grow as haze forming compounds were added to the atmosphere. To assess this effect on the early Earth we assume several plausible scenarios, shown in Fig. 5. First, as a baseline we consider no antigreenhouse effect, $\gamma=0$. Next we consider a constant antigreenhouse effect equal to that on Titan at the present time, $\gamma=4 / 7$. The Titan-like case requires more than twice the infrared opacity to reach the freezing point when compared with the case without the antigreenhouse effect.

There are several reasons why the Titan-like case may be too optimistic for the early Earth. First, Titan's haze is opaque in the ultraviolet but fairly transparent in the visible compared to other abiotic organics (Khare et al. 1984, 1994, Sagan et al. 1992). 


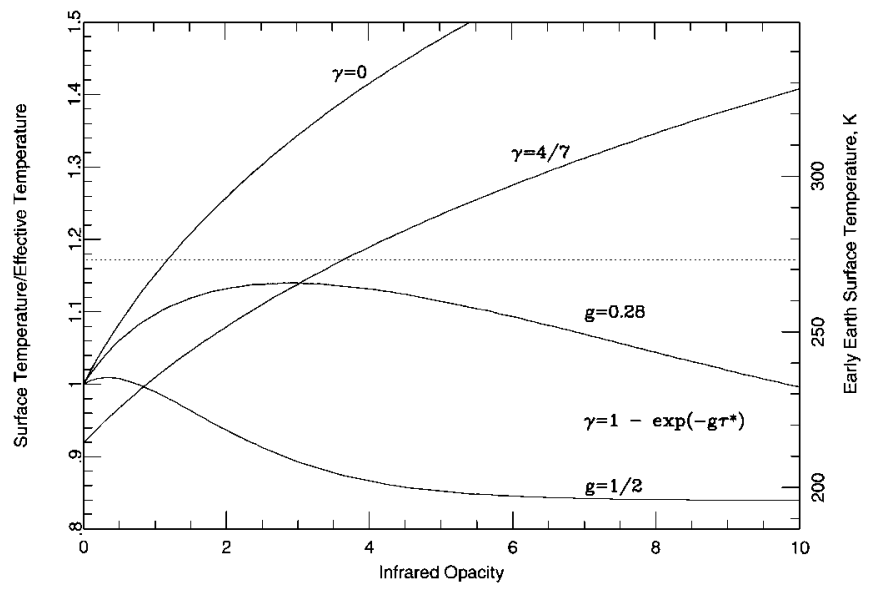

FIG. 5. Surface temperature ratioed to effective temperature as a function of infrared opacity for a grey greenhouse atmosphere with the antigreenhouse parameter $(\gamma)$ determined in various ways. The surface temperature for the early Earth is shown on the right axis - the freezing point is indicated with the dotted line. Titan's present antigreenhouse layer has a value of $\gamma=4 / 7$.

Thus organics produced on the early Earth are likely to have values of optical properties such that more of the visible radiation is blocked corresponding to larger values of $\gamma$. Second, it is unlikely that $\gamma$ is constant with time. On Titan only $2 \%$ of the photolysis products of $\mathrm{CH}_{4}$, and from 10 to $100 \%$ of the $\mathrm{N}$ from $\mathrm{N}_{2}$ dissociation, end up in the haze particles (McKay 1996). Changes in atmospheric chemistry could significantly alter the rate of solid organic production. Inclusion of $\mathrm{NH}_{3}$, which readily reacts to form solids, would also be expected to increase haze production. At low levels of $\mathrm{NH}_{3}$, it is reasonable to expect that the haze production would increase with increasing $\mathrm{NH}_{3}$. Thus, the more $\mathrm{CH}_{4}$ and $\mathrm{NH}_{3}$ the thicker the haze and the stronger the antigreenhouse effect will be. For this case we assume that the amount of sunlight that escapes the antigreenhouse layer and reaches the lower atmosphere declines exponentially as the haze becomes thicker and that the haze is proportional to the infrared opacity of the haze forming gases. Thus the antigreenhouse effect can be written as $\gamma=1-\exp \left(-g \tau^{*}\right)$, where $g$ is the constant that depends on the optical properties (indices of refraction) of the haze layer and the relation between haze and infrared opacity. We consider first a case that matches the present Titan; $\gamma=4 / 7, \tau^{*}=3$, and thus $g=0.28$. In this case the antigreenhouse effect triumphs and the temperature on the early Earth never reaches the freezing point. Darker hazes $(g=1 / 2)$ produce even less warming and at high opacity the antigreenhouse effect becomes complete and the surface temperature goes asymptotically to the antigreenhouse limit, $T_{\mathrm{e}} / \sqrt[4]{2}$. The temperature just reaches freezing for a value of $g=0.23$. The cooling effect of an antigreenhouse layer could be altered if this layer was within the convective part of the troposphere. However, for both Titan and a reducing early Earth the pressure at which the haze forms is expected to be $10^{-4}$ bars or less (McKay et al. 1989).

The antigreenhouse effect is an important feature of planetary atmospheres and the analytical solutions we have developed pro- vide a convenient way to estimate the combined greenhouse and antigreenhouse effects. While Titan has the only known significant antigreenhouse effect, this effect is suggested to have played an important role on the Earth in the past and, through scenarios for nuclear winter (Turco et al. 1983), defines a future for the Earth we seek to avoid.

\section{APPENDIX. ANTIGREENHOUSE EQUATION}

Milne (1922) and Goody and Yung (1989) show that the solution for the greenhouse effect using the two-stream gray approximation with heating only at the surface is

$$
\sigma T^{4}(\tau)=F_{\mathrm{s}}\left(\frac{1}{2}+\frac{3}{4} \tau\right)
$$

and for the surface temperature

$$
\sigma T_{\mathrm{s}}^{4}=F_{\mathrm{s}}\left(1+\frac{3}{4} \tau^{*}\right)
$$

and the solution for heating due to uniform exponential absorption of the solar beam is

$$
\sigma T^{4}(\tau)=F_{\mathrm{s}}\left[\frac{1}{2}+\frac{3}{4 k}+\left(\frac{k}{4}-\frac{3}{4 k}\right) \mathrm{e}^{-k \tau}\right]
$$

and it can be shown that the surface temperature is given by

$$
\sigma T_{\mathrm{s}}^{4}=F_{\mathrm{s}}\left[\frac{1}{2}+\frac{3}{4 k}+\left(\frac{1}{2}-\frac{3}{4 k}\right) \mathrm{e}^{-k \tau^{*}}\right],
$$

where $F_{\mathrm{S}}$ is the globally averaged sunlight absorbed by the planet, $\tau$ is the infrared opacity, $\tau^{*}$ is the total column infrared opacity, and $k$ is the ratio of the solar extinction coefficient to the infrared absorption coefficient, $k=\tau_{\mathrm{v}} / \tau$.

It is readily shown that due to the linearity of the basic equations the solution in the case of both heating at the surface and extinction of flux in the atmosphere is just the sum of the solutions in Eqs. (5) and (3). We can in general write

$$
\begin{aligned}
\sigma T^{4}(\tau)= & \left(F_{\mathrm{g}}+(1-\gamma) F_{\mathrm{s}}\right)\left(\frac{1}{2}+\frac{3}{4} \tau\right) \\
& +\gamma F_{\mathrm{s}}\left[\frac{1}{2}+\frac{3}{4 k}+\left(\frac{k}{4}-\frac{3}{4 k}\right) \mathrm{e}^{-k \tau}\right]
\end{aligned}
$$

and the surface temperature is

$$
\begin{aligned}
\sigma T_{\mathrm{s}}^{4}= & \left(F_{\mathrm{g}}+(1-\gamma) F_{\mathrm{s}}\right)\left(1+\frac{3}{4} \tau^{*}\right) \\
& +\gamma F_{\mathrm{s}}\left[\frac{1}{2}+\frac{3}{4 k}+\left(\frac{1}{2}-\frac{3}{4 k}\right) \mathrm{e}^{-k \tau^{*}}\right],
\end{aligned}
$$

where $\gamma$ is the factor expressing the strength of the antigreenhouse effect, $(1-\gamma) F_{\mathrm{s}}$ is the fraction of solar flux that is directly deposited in the lower atmosphere and surface, $\gamma F_{\mathrm{s}}$ is the solar flux that is being absorbed in the antigreenhouse layer, and $F_{\mathrm{g}}$ represents any additional nonsolar heating terms at the surface.

We can simplify the antigreenhouse equation even further. First we take the limit of Eq. (8) when $k \rightarrow \infty$, this gives

$$
\sigma T_{\mathrm{s}}^{4}=\left(F_{\mathrm{g}}+(1-\gamma) F_{\mathrm{s}}\right)\left(1+\frac{3}{4} \tau^{*}\right)+\frac{\gamma F_{\mathrm{s}}}{2} .
$$

We note that $k$ does not appear in Eq. (9). This equation is therefore a good approximation to the antigreenhouse effect due to a haze layer that efficiently blocks a fraction of the sunlight from reaching the surface and represents a simple way to incorporate the antigreenhouse effect into an analytic expression. The assumption that the ratio of visible to infrared absorption is constant drops out of the analysis - as long as the absorption due to the antigreenhouse layer is very large in certain wavelengths (usually the ultraviolet and blue) and negligible in others (usually the red and infrared). 
Two limits can be readily considered for Eq. (9). First when $\gamma=0$, no antigreenhouse effect, the greenhouse effect is recovered. The other limit that is interesting is the case in which $\gamma \rightarrow 1$ and the antigreenhouse effect is complete. In this case the surface temperature remains at the value $T_{\mathrm{e}} / \sqrt[4]{2}$, even as $\tau^{*} \rightarrow \infty$, which is the expected antigreenhouse limit (McKay et al. 1991).

\section{ACKNOWLEDGMENTS}

This research was supported by subventions from the NASA Planetary Atmospheres Program. RDL acknowledges the support of the Cassini project.

\section{REFERENCES}

Courtin, R., D. Gautier, and C. P. McKay 1995. Titan's thermal emission spectrum: Re-analysis of the Voyager infrared measurements. Icarus 114, 144-162.

Goody, R. M., and Y. L. Yung 1989. Atmospheric Radiation: Theoretical Basis, Oxford Univ. Press, Oxford.

Khare, B. N., C. Sagan, E. T. Arakawa, F. Suits, T. A. Callcott, and M. W. Williams 1984. Optical constants of organic tholins produced in a simulated titanian atmosphere: From soft X-ray to microwave frequencies. Icarus $\mathbf{6 0}$, 127-137.

Khare, B. N., C. Sagan, W. R. Thompson, E. T. Arakawa, C. Meisse, and P. S. Tuminello 1994. Optical properties of poly-HCN and their astronomical applications. Can. J. Chem. 72, 678-694.

Lellouch, E., A. Coustenis, D. Gautier, F. Raulin, N. Dubouloz, and C. Frere 1989. Titan's atmosphere and hypothesized ocean: A reanalysis of the Voyager 1 radio-occultation and IRIS $7.7 \mu \mathrm{m}$ data. Icarus 79, 328-349.
Lorenz, R. D., J. I. Lunine, and C. P. McKay 1997b. Titan under a red giant sun: A new kind of "habitable" world. Geophys. Res. Let. 24, 2905-2908.

Lorenz, R. D., C. P. McKay, and J. I. Lunine 1997a. Photochemically-induced collapse of Titan's atmosphere. Science 275, 642-644.

McKay, C. P. 1996. Elemental composition, solubility, and optical properties of Titan's organic haze. Planet. Space Sci. 44, 741-747.

McKay, C. P., S. C. Martin, C. A. Griffith, and R. M. Keller 1997. Temperature lapse rate and methane in Titan's troposphere. Icarus 129, 498-505.

McKay, C. P., J. B. Pollack, and R. Courtin 1989. The thermal structure of Titan's atmosphere. Icarus 80, 25-53.

McKay, C. P., J. B. Pollack, and R. Courtin 1991. The greenhouse and antigreenhouse effects on Titan. Science 253, 1118-1121.

McKay, C. P., J. B. Pollack, J. I. Lunine, and R. Courtin 1993. Coupled atmosphere-ocean models of Titan's past. Icarus 102, 88-98.

Milne, E. A. 1922. Radiative equilibrium, the insolation of the atmosphere. Phil. Mag. 144, 872.

Sagan, C., and C. Chyba 1997. The early faint sun paradox: Organic shielding of ultraviolet-labile greenhouse gases. Science 276, 1217-1221.

Sagan, C., W. R. Thompson, and B. N. Khare 1992. Titan: A laboratory for prebiological organic chemistry. Acc. Chem. Res. 25, 286-292.

Samuelson, R. E. 1983. A radiative equilibrium model of Titan's atmosphere. Icarus 53, 364-387.

Samuelson, R. E., N. R. Neth, and A. Borysow 1997. Gaseous abundances and methane supersaturation in Titan's troposphere. Planet. Space Sci. 45, 959980.

Turco, R. P., O. B. Toon, T. P. Ackerman, J. B. Pollack, and C. Sagan 1983. Nuclear winter: Global consequences of multiple nuclear explosions. Science 222, 1283-1292. 